\title{
Current antimicrobial sensitivity pattern of typhoidal salmonellae in a referral diagnostic centre
}

\author{
Umer Shujat, Aamer Ikram, Inam Qadir Javaid Hashmi, Shahid Ahmed Abbasi, \\ Amna Afzal, Muhammad Ayyub
}

Armed Forces Institute of Pathology, Rawalpindi, Pakistan

\section{Summary}

Background: Infections caused by typhoidal salmonellae are an important public health concern in Pakistan. Inappropriate and injudicious use of fluoroquinolones has reduced their efficacy due to development of high level resistance.

Aim: To ascertain the current susceptibility pattern of typhoidal salmonellae thus guiding the physicians for better management of typhoid patients.

Materials and Methods: A study was conducted at our institution from January 2012 through December 2013 to investigate current susceptibility pattern of typhoidal salmonellae.

Results: Out of 200 isolates, 107 (53.5\%) were identified as Salmonella Typhi and 93 (46.5\%) as Salmonella Paratyphi A. Sensitivities of Salmonella Typhi were as follows: ampicillin (48.6\%), chloramphenicol (45.8\%), co-trimoxazole (40.1\%), ciprofloxacin (11.2\%). Sensitivities of Salmonella Paratyphi A were: ampicillin (80.6\%), chloramphenicol (89.2\%), co-trimoxazole (90.3\%), and ciprofloxacin (16.1\%). No resistance was detected against third generation cephalosporins.

Correspondence: Umer Shujat, Department of Microbiology, Armed Forces Institute of Pathology, Rawalpindi 46000, Pakistan.

Tel.: +92.3335112041.

E-mail: umershujaat2144@gmail.com

Key words: Antimicrobial sensitivity; enteric fever; fluoroquinolones; Salmonella Typhi; Salmonella Paratyphi A.

Contributions: US, AI, SAA made substantial contribution to conception, design of work, analysis and drafting; IQJH and AA made valuable contributions in collection of data; MA revised it critically and made final approval of the version to be submitted for publication.

Conflict of interest: the authors declare no potential conflict of interest.

Received for publication: 15 August 2014.

Revision received: 26 June 2015.

Accepted for publication: 25 November 2015.

(C) Copyright U. Shujat et al., 2016

Licensee PAGEPress, Italy

Microbiologia Medica 2016; 31:4669

doi:10.4081/mm.2016.4669

This article is distributed under the terms of the Creative Commons Attribution Noncommercial License (by-nc 4.0) which permits any noncommercial use, distribution, and reproduction in any medium, provided the original author(s) and source are credited.
Conclusions: Typhoidal salmonellae are still entirely susceptible to third generation cephalosporins in our setting. Marked rise in resistance to fluoroquinolones has reduced their empirical usage. Sensitivity of Salmonella Paratyphi A to conventional antityphoid drugs was encouraging.

\section{Introduction}

Infections caused by typhoidal Salmonellae are an important public health concern worldwide. The World Health Organization (WHO) has estimated that annually 21.7 million people contract typhoid fever out of which 217,000 succumb to the disease. Paratyphoid fever, usually caused by Salmonella Paratyphi A and occasionally by Salmonella Paratyphi B and C, accounts for 5.4 million new cases worldwide each year (10). Indiscriminate use of antibiotics during the previous century rendered $S$. Typhi and $S$. Paratyphi A resistant to the conventional antityphoids like ampicillin, chloramphenicol and co-trimoxazole. Resistance to newer drugs like ciprofloxacin soon followed; initially with development of low level resistance and lastly of high level resistance in typhoid endemic countries $(7,10,11)$. This has narrowed down the therapeutic options to third generation cephalosporins, as resistance to these antibiotics has been reported less frequently. In the last few years, azithromycin has also been used to treat uncomplicated typhoid fever and several clinical and in vitro studies have demonstrated its efficacy against typhoidal salmonellae $(3,6,9)$. Recent studies have shown reemerging susceptibility to conventional drugs previously rendered useless by excessive resistance $(5,13)$.

This study was carried out to evaluate the current susceptibility pattern of typhoidal salmonellae against antityphoidal drugs and to provide physicians with a guideline for the proper choice of antibiotics against infections by these bacteria.

\section{Materials and Methods}

This study was conducted at the Armed Forces Institute of Pathology, Rawalpindi, Pakistan from January 2012 through December 2013. By using the BACTEC 9120 (Becton, Dickinson and Company, Franklin Lakes NJ, USA) continuous monitoring blood culture system and bottles provided by the manufacturer consecutive, non-duplicate typhoidal salmonellae were included in the study. Isolates were identified by colony characteristics on 5\% sheep blood (Oxoid, Basingstoke, UK) and MacConkey agar (Oxoid), biochemical reactions on API 20E (bioMerieux, Marcy-l'Étoile, France) and specific Salmonella antisera (Denka Seiken, Tokyo, Japan). Antibiotic susceptibility testing was carried out on Mueller-Hinton agar (Oxoid, UK) by the Kirby-Bauer 
disc diffusion method; incubating plates aerobically at $35^{\circ} \mathrm{C} \pm 2$ for 18 24 hours and interpreting inhibition zones according to Clinical and Laboratory Standards Institute (CLSI) guidelines (4). Antibiotic discs (Oxoid, UK) used were ampicillin (10 $\mu \mathrm{g})$, chloramphenicol $(30 \mu \mathrm{g})$, trimethoprim-sulfamethoxazole $(1.25 / 23.75 \mu \mathrm{g})$, nalidixic acid $(30 \mu \mathrm{g})$, ceftriaxone $(30 \mu \mathrm{g})$ and cefixime $(5 \mu \mathrm{g})$. Salmonella Typhi ATCC 700931 and Salmonella Paratyphi A ATCC 9150 were used as controls.

\section{Results}

During the study period 200 isolates of Salmonella Typhi and Salmonella Paratyphi A were dealt in the department. The typhoidal isolates were subjected to susceptibility testing against conventional and newer antityphoid drugs.

The mean age of patients was 19 years (range 4 to 66 years); 126 (63\%) patients were males. One hundred and seven isolates (53.5\%) were identified as Salmonella Typhi and 93 (46.5\%) as Salmonella Paratyphi A.

Sensitivity of Salmonella Typhi isolates to various antimicrobials tested was: ampicillin 48.6\%, chloramphenicol 45.8\%, co-trimoxazole $40.1 \%$, nalidixic acid $8.4 \%$, ciprofloxacin $11.2 \%$, ceftriaxone and cefixime 100\%. The sensitivity pattern of Salmonella Paratyphi A was as follows: ampicillin $80.6 \%$, chloramphenicol $89.2 \%$, co-trimoxazole $90.3 \%$, nalidixic acid $9.7 \%$, ciprofloxacin $16.1 \%$, ceftriaxone $100 \%$ and cefixime $100 \%$.

\section{Discussion}

Typhoid fever is a common cause of bloodstream infections in developing countries like Pakistan. This is mainly due to poor sanitation conditions, unsafe drinking water supply and inadequate surveillance systems for food borne infections (13). Salmonella Typhi and Salmonella Paratyphi A are more prevalent in our country whereas infections caused by serotypes Paratyphi B or C are rare $(1,15)$.

In the present study, males were found to be more prone to contracting typhoid fever probably as a result of outdoor working and eating and drinking food from street vendors. Similar statistics have been regionally reported by Gupta et al. from India and by Karkey et al. from Nepal $(6,10)$.

Among typhoidal salmonellae, $S$. Typhi was more common than $S$. Paratyphi A. $S$. Typhi is still the leading typhoid Salmonella, but the frequency of Paratyphi A isolates has significantly increased over the past years, as observed by various authors $(8,15)$. Typhoid vaccines (Vi polysaccharide and live oral Ty21a) have been used extensively in our country in the recent years. As these preparations do not provide protection against $S$. Paratyphi A, this could have lead to relative rise in Paratyphi A infection rates (16).

In our study, sensitivities of $S$. Typhi and $S$. Paratyphi A to ciprofloxacin were low. This could partially be due to revision of zone interpretation criteria of CLSI (document M100; 2013) from $21 \mathrm{~mm}$ to $31 \mathrm{~mm}$ for being susceptible, thus classifying the majority of our isolates as intermediately resistant. A study from Karachi, Pakistan, reported high sensitivity of typhoidal salmonellae to ciprofloxacin (96.4\%), however, the zone diameters were interpreted according to previous CLSI guidelines (1). Similarly, a study conducted by Raza et al from Nepal showed excellent sensitivity to ciprofloxacin for $S$. Typhi and $S$. Paratyphi A isolates (15).

Kumar et al. from India showed high sensitivity of salmonellae to conventional antityphoid drugs, such as chloramphenicol (95.3\%), ampicillin (94.5\%) and co-trimoxazole (94.5\%) (12). In comparison, much lower susceptibilities were seen in our study. Lakshmi et al. reported increasing sensitivity of chloramphenicol against both $S$. Typhi and $S$. Paratyphi A and similar trend was reported by another Indian study $(12,13)$. The sensitivities of $S$. Paratyphi A isolates in our study to chloramphenicol, ampicillin and co-trimoxazole were similar to a study conducted by Gupta et al. from India (6).

The sensitivity results of typhoidal salmonellae to conventional antityphoid drugs, though encouraging, are not sufficient to re-employ these drugs as empirical treatment. Resistance against third generation cephalosporins is still uncommon with only sporadic cases reported from Bangladesh and India (17). All isolates of $S$. Typhi and $S$. Paratyphi in our study were sensitive to ceftriaxone and cefixime. Other regional studies also reported excellent sensitivity to ceftriaxone in $S$. Typhi $(2,15)$.

\section{Conclusions}

Among typhoidal salmonellae, a notable proportion of $S$. Paratyphi A isolates in our setting was susceptible to conventional antityphoid drugs. The increase in resistance to fluoroquinolones has rendered them unsuitable for empirical treatment of typhoid fever and the susceptibility profile should be interpreted according to latest guidelines. Resistance to third generation cephalosporins was not detected but injudicious use may lead to this menace in the near future.

\section{References}

1. Abdullah FE, Haider F, Fatima K, et al. Enteric fever in Karachi: current antibiotic susceptibility of Salmonella isolates. J Coll Physician Surg Pak 2012;22:147-50.

2. Butt T, Ahmad RN, Salman M, et al. Changing trends in drug resistance among typhoidal salmonellae in Rawalpindi, Pakistan. East Mediterr Health J 2005;11:1038-44.

3. Capoor MR, Nair D. Quinolone and cephalosporin resistance in enteric fever. J Glob Infect Dis 2010;2:258-26.

4. Clinical and Laboratory Standards Institute. Performance standards for antimicrobial susceptibility testing; twenty third informational supplement M100-S23. Wayne: CLSI; 2013.

5. Gokul BN, Menezes GA, Harish BN. ACC-1 beta-lactamase producing salmonella enterica serovar Typhi, India. Emerg Infect Dis 2010;16:1170-71.

6. Gupta V, Singla N, Bansal N, et al. Trends in antibiotic resistance patterns of enteric fever isolates - a three year report from a tertiary care centre. Malays J Med Sci 2013;20:71-5.

7. Harish BN, Menezes GA, Sarangapani K, et al. A case report and review of literature: Ciprofloxacin resistant Salmonella enterica serovar Typhi in India. J Infect Devel Countries 2008;2:324-32.

8. Harish BN, Menezes GA. Antimicrobial resistance in typhoidal salmonellae. Indian J Med Microbiol 2011;29:223-9.

9. Hassing RJ, Goessens WH, van Pelt W, et al. Salmonella subtypes with increased MICs for azithromycin in travellers returned to The Netherlands. Emerg Infect Dis 2014;20:705-8.

10. Karkey A, Thompson CN, Tran Vu Thieu N, et al. Differential epidemiology of salmonella typhi and paratyphi A in Kathmandu, Nepal: a matched case control investigation in a highly endemic enteric fever setting. PLoS Negl Trop Dis 2013;7:e2391.

11. Krishnan P, Stalin M, Balasubramanian S. Changing trends in antimicrobial resistance of Salmonella enterica serovar TYPHI and Salmonella enterica serovar paratyphi A in Chennai. Indian J Pathol Microbiol 2009;52:505-8.

12. Kumar Y, Sharma A, Mani KR. Re-emergence of susceptibility to 
conventionally used drugs among strains of Salmonella Typhi in central west India. J Infect Dev Ctries 2011;5:227-30.

13. Lakshmi V, Ashok R, Susmita J, et al. Changing trends in the antibiograms of Salmonella isolates at tertiary care hospital in Hyderabad. Indian J Med Microbiol 2006;24:45-8.

14. Qaiser S, Irfan S, Khan E, et al. In vitro susceptibility of typhoidal Salmonellae against newer antimicrobial agents: a search for alternate treatment options. J Pak Med Assoc 2011;61:462-5.
15. Raza S, Tamrakar R, Bhatt CP, et al. Antimicrobial susceptibility patterns of Salmonella Typhi and Salmonella Paratyphi A in a tertiary care hospital. J Nepal Health Res Counc 2012;10:214-7.

16. Sahastrabuddhe $S$, Carbis $R$, Wierzba TF, et al. Increasing rate of salmonella paratyphi A and current status of its vaccine development. Expert Rev Vaccines 2013;12:1021-31.

17. Saha SK, Talukder SY, Islam M, et al. A highly ceftriaxone resistant Salmonella Typhi in Bangladesh. Pediatr Infect Dis J 1999;18:387. 